\title{
De la vocation féminine à l'expertise féministe : essai sur l'évolution du service social au Québec (1939-1990)
}

\section{L.-H. Groulx}

Volume 49, numéro 3, hiver 1996

URI : https://id.erudit.org/iderudit/305447ar

DOI : https://doi.org/10.7202/305447ar

Aller au sommaire du numéro

Éditeur(s)

Institut d'histoire de l'Amérique française

ISSN

0035-2357 (imprimé)

1492-1383 (numérique)

Découvrir la revue

Citer cet article

Groulx, L.-H. (1996). De la vocation féminine à l'expertise féministe : essai sur l'évolution du service social au Québec (1939-1990). Revue d'histoire de l'Amérique française, 49(3), 357-394. https://doi.org/10.7202/305447ar
Résumé de l'article

Cet article expose trois principales figures identitaires du service social soit féminine et vocationnelle, professionnelle et féministe. Cette prise en compte de la pluralité des identités amène l'auteur à s'interroger sur l'interprétation dominante en termes de professionnalisation/déprofessionnalisation et sur le sens à donner à ce travail symbolique sur l'image sociale du métier. 


\title{
DE LA VOCATION FÉMININE À L'EXPERTISE FÉMINISTE: ESSAI SUR L'ÉVOLUTION DU SERVICE SOCIAL AU QUÉBEC (1939-1990)
}

\author{
L.-H. GROULX \\ École de service social \\ Université de Montréal
}

\section{RÉSUMÉ}

Cet article expose trois principales figures identitaires du service social soit féminine et vocationnelle, professionnelle et féministe. Cette prise en compte de la pluralité des identités amène l'auteur à s'interroger sur l'interprétation dominante en termes de professionnalisation/déprofessionnalisation et sur le sens à donner à ce travail symbolique sur l'image sociale du métier.

\begin{abstract}
This article presents the three main social images that have been used to characterize social work in Quebec. These images are: a calling to traditional woman's value, the professional value and the feminist attitude. It is by taking into account the plurality of these identities that the author questions the main interpretation of the evolution of social work in terms of professionnalisation/ déprofessionnalisation and presents an alternative analysis of these changes.
\end{abstract}

\section{INTRODUCTION}

La problématique professionnelle reste au cœur des interrogations sur le service social et son évolution, que celle-ci soit formulée en termes de professionnalisation ou de déprofessionnalisation. Les analyses des années 1960 et 1970 se sont interrogées sur la difficile intégration du service social, défini comme moderne et professionnel, à un milieu canadien-français perçu comme traditionnel et conservateur. Cette perception qui renvoie à la thèse du retard professionnel, situe la naissance et l'émergence du service social comme un apport extérieur au milieu socioculturel d'accueil. L'histoire devient alors le récit de sa difficile professionnalisation. Les analyses des années 1980 ont inversé, pourrait-on dire, les termes de l'interrogation 
en posant moins la question des problèmes de l'intégration du service social dans la société mais plutôt celle des effets du développement de la société capitaliste ou technocratique sur le service social professionnel, entraînant sa déprofessionnalisation ou son éclatement.

Malgré la différence de ces analyses sociohistoriques, le service social est constamment pensé comme une entité, définie à partir d'attributs professionnels particuliers, correspondant à une culture, une rationalité, une compétence méthodologique ou une autonomie d'action. Selon Rocher, c'est cette culture professionnelle qui rend, dans les années 1940 et 1950, son intégration difficile à cause d'une mentalité traditionnelle, religieuse, cléricale, dominante dans le milieu canadien-français. C'est en outre ce même «univers professionnel spécifique» qui éclate, selon Renaud, dans les années 1980, sous la pression de la nouvelle division du travail et de la nouvelle rationalité technocratique. L'histoire du service social ainsi présentée et mise en œuvre «reproduit, dans la démarche qui se veut de connaissance, l'idéologie du groupe qu'il étudie ${ }^{1} »$, car les analyses deviennent alors le récit des étapes, des phases et des péripéties de cette lutte du service social pour sa reconnaissance ou son affirmation professionnelle. Celui-ci fait aussi figure, dans ces analyses, d'acteur ou de héros aux prises avec des forces sociales qui s'opposent ou se présentent comme des obstacles à son identité ou à son projet professionnel. Ces forces sociales adverses prennent alternativement les noms de mentalité cléricale ou religieuse, de pratiques sociales dominantes ou de rationnalité technocratique ou de rapports sociaux capitalistes. Le service social conserve, pourrait-on dire, la même entité ou essence tout au long de son histoire et devient alors ennobli par et dans son histoire. L'histoire est alors convoquée pour dire et fonder le service social.

Pour être efficace, l'analyse se doit de s'arracher ou rompre avec cette perspective ou cette vision homogène du monde social ${ }^{2}$ et de refuser toute démarche téléologique où tout concourt au maintien ou au développement d'une définition a priori ou à la réalisation d'un modèle professionnel de référence ${ }^{3}$ qui agit comme code organisateur

1. R. Chartier, «Préface», N. Elias, La société de cour (Paris, Flammarion, 1985).

2. Cette vision homogène du monde social se construit à partir d'une visée fonctionnaliste des groupes professionnels où l'on «éternise un ensemble de traits différentiels sans s'interroger sur les conditions et donc les limites de leur pertinence». L. Karpik, Les avocats (Paris, Flammarion, 1995), 16. L'identité des groupes professionnels est tenue pour acquise et le travail de recherche ne vise qu'à retrouver ou confirmer la définition implicite préalablement acceptée.

3. Ces analyses se développent à partir d'une vue rétrospective de l'histoire où l'on réinterroge les faits passés à partir du présent. Le sens des événements passés changent de signification car il devient décontextualisé à partir des événements présents. Notre démarche 
d'interprétation et de construction du récit historique. On est amené à refuser de situer le service social dans un rapport d'extériorité par rapport à la société et à faire le pari méthodologique que l'histoire du service social est aussi l'histoire de la société à laquelle il appartient. D'où l'hypothèse d'une pluralité d'identités en service social, exprimant des normes d'action variables selon les périodes historiques et les champs d'action sociale où il est inséré. Cette option pour la discontinuité permet de repérer une diversité de pratiques, une variété de formes d'action, une pluralité de figures du service social, car la pleine compréhension de son histoire oblige à prendre en compte ses redéfinitions successives qui font partie intégrante de son histoire.

Notre démarche repose sur l'exploitation de trois types de sources d'information. Nous avons dépouillé les archives de l'Ecole de service social de Montréal, de l'Archevêché de Montréal et de la Fédération des œuvres de charité canadiennes-françaises. Nous avons procédé à l'examen détaillé d'un échantillon de comptes rendus d'admission de l'École de Montréal ${ }^{4}$. Nous l'avons complété par l'étude d'une documentation écrite qui comprenait imprimés, brochures, livres, rapports et thèses couvrant les périodes étudiées. Nous nous sommes enfin entretenu avec quatre générations de travailleurs sociaux. Le croisement des témoignages oraux avec les matériaux d'archives et la documentation publique permet, par une sorte de triangulation des données, de mieux évaluer l'évidence documentaire et de faire une critique plus judicieuse des sources. Il faut cependant souligner que notre priorité réside moins dans l'examen de la vérité des sources à l'étude, mais procède plutôt, comme le note Duby, de la nécessité de faire ressortir, à partir de ces mêmes sources, les représentations que le groupe social se construit subjectivement de lui-même ${ }^{5}$.

s'est construite à partir de trois refus: refus de cerner l'identité du service social en termes d'essence, refus de penser l'histoire comme l'évolution ou la réalisation de cette essence et refus de situer le service social dans un rapport d'extériorité par rapport à la société ou aux groupes sociaux.

4. Les entrevues d'admission constituaient, durant les années 1940 jusqu'aux années 1970, une des conditions d'admission à la formation universitaire en service social et étaient réalisées, non seulement par des membres du personnel enseignant mais aussi par des praticiens sociaux, en particulier par des surveillants de stages. Elles portaient sur l'évaluation de la personnalité des candidats à la formation et demeurent pour cette raison un matériel privilégié pour l'étude des valeurs en service social. Elles permettent, de ce fait, d'en cerner l'existence et l'évolution à partir de la définition socialement valorisée d'un bon et d'un mauvais candidat. Comme ces documents ont été rédigés sous le couvercle de la confidentialité et portent sur des personnes encore vivantes, la diffusion de ce matériel pose plusieurs questions d'éthiques. Nous avons omis, volontairement, de spécifier l'année exacte du compte rendu et avons été attentif dans nos citations à éliminer tout indice qui pourrait faire reconnaître des candidats(es) ou des évaluateurs(trices). Le même souci de confidentialité nous a guidé dans le compte rendu de nos matériaux d'entrevues.

5. G. Duby, L'histoire continue (Paris, Jacob, 1991). 
Nous avons divisé notre texte en deux parties afin de présenter respectivement les résultats de notre recherche et d'en dégager des pistes d'interprétation. Dans la première partie, nous exposons les trois principales figures identitaires du service social soit féminine et vocationnelle, professionnelle et féministe. Nous présentons dans une deuxième partie, des interprétations et réflexions sociohistoriques sur le développement du service social en discutant du sens que l'on peut dégager de la variation et de la pluralité des identités sociales du service social, car notre visée a été principalement de comprendre la réalité sociale du service social «par le dedans en reconstituant, pour chaque période, les catégories dans lesquelles elle était pensée ${ }^{6}{ }^{6}$.

\section{I - L'IMPORTANCE DE LA VOCATION}

Les premières manifestations du service social au Canada français apparaissent à Montréal au début des années 1930, grâce à l'initiative conjointe et conjuguée de l'Archevêché de Montréal et d'hommes d'affaires canadiens-français qui, en 1932, décident de mettre sur pied une Fédération des Euvres de Charité pour centraliser les demandes de fonds et assurer un financement aux œuvres sociales existantes. Cette nouvelle fédération créa, en 1935, le Conseil des Euvres afin d'assurer la coordination et la planification des œuvres sociales.

Puis en 1938, en collaboration avec l'Archevêché, fut fondée la première agence de service social, le Bureau d'aide aux familles, pour répondre aux demandes de secours qui «affluaient à l'Archevêché» et à la «Fédération» et que les œuvres traditionnelles de charité ne pouvaient gérer. Pour assurer une main-d'œuvre adéquate pour ses premiers services sociaux, une école universitaire de service social fut mise sur pied en 1939. Nous retrouvons alors au début des années 1940 les principales conditions institutionnelles, tant au niveau des sources de financement, du marché du travail que de la formation, pour assurer et garantir le développernent et l'implantation du service social dans le milieu canadien-français.

Ce service social pratiqué par des auxiliaires sociales se définissait comme vocationnel, pratique et féminin tout en s'intégrant au catholicisme social de l'époque.

Cette idée vocationnelle du service social transcendait les seules exigences d'un métier ou d'une profession. Comme l'exprime le premier directeur de l'École de service social de Montréal: «Le service social s'il est une profession, est surtout une vocation. Il est donc

6. A. Burguière, «Le changement social: brève histoire d'un concept», B. Lepetit, dir., Les formes de l'expérience (Paris, Albin Michel, 1995). 
nécessaire d'être résolu de se dépenser pour les autres, bien plus que d'attendre un profit personnel de la science et de l'expérience qu'on vient puiser à l'École? ${ }^{7}$ »

La plupart des auxiliaires sociales percevaient et interprétaient spontanément leur choix sur le registre de la vocation. Ces témoignages de candidates à l'admission sont éloquents à cet égard ${ }^{8}$.

Si je n'avais aucun goût pour la vocation d'auxiliaire sociale, je resterais en psychologie, c'est avec joie et sérénité d'esprit que j'entreprends mon service social. Contribuer à l'épanouissement de la vie sociale et à l'apothéose de la personne humaine en apportant aux hommes un bien-être soit physique, soit moral ou parfois même spirituel, ce sont là des motifs de haute altitude qui m'incitent à entreprendre des études en service social. Le motif qui me pousse au service social, c'est d'abord un appel qui se fait pressant en moi, celui de servir. Après avoir considéré mes goûts et aptitudes et après avoir prié, j'ai pensé que ma place à moi pour «servir» était au service social, mon idéal a toujours été de soulager les déshérités de la vie, quels qu'ils soient, et je crois que je pourrai réaliser mon idéal en embrassant la carrière d'auxiliaire sociale ${ }^{9}$.

Le service social se réalise dans l'accomplissement d'activités pratiques, dans la réponse à des demandes urgentes, dans la distribution de ressources et la mise sur pied de services concrets. Précisons que le gouvernement provincial, en reconnaissant en 1940 les agences sociales comme des institutions d'assistance publique «sans mur», autorisait les auxiliaires sociales à fournir des prestations en espèces aux indigents ${ }^{10}$. Cette mesure est importante sous deux aspects: elle assure un certain financement des agences supportant ainsi leur développement et, par le fait même, les reconnaît et leur donne une légitimité en tant qu'organisme d'assistance.

7. Prospectus et programme, École de service social, Montréal, 1942.

8. Ce thème vocationnel prend parfois des accents lyriques comme chez cette étudiante qui écrivait à la même époque dans le journal étudiant: «Mais toujours le grand souffle «social» gonflera le mouvement de nos voiles. Il restera l'âme, l'inspiration, l'élément formel, pétrissant notre vie [...] C'est que nous entreprenons une vie de rudesse et de peines. Nous y laisserons le meilleur de notre cœur, de nos énergies vitales. L'appel à notre profession a toute l'austère douceur de l'appel à la vocation. Il est tout aussi irréversible. Il comporte tous les dévouements et pourra parfois exiger le don total. Nous n'avons déjà plus le droit de nous dérober à la fatigue, aux renoncements, à la besogne [...] Travailler pour nous, c'est exercer une action formatrice sur les relations humaines et les forces sociales.» Serso, journal étudiant de l'École de service social (Montréal, 1945), 4.

9. Serso, op. cit., 2.

10. N. Zay, "Assistance publique et service social», Service social, 10,3 (1961): 64-85. 
La formation se voulait d'abord pratique et les conditions étaient des garanties d'une bonne santé, une attestation de la valeur morale ainsi que des études secondaires terminées, car «la formation en service social s'adressait à toute personne qui désirait faire le bien et bien le faire». Cette vocation à faire le bien était perçue et définie comme une activité proprement féminine ${ }^{11}$. Ainsi la directrice des études de la section service social de Montréal déclarait, dans un journal local, que les travailleuses sociales étaient choisies pour «leur sentiment fémi$\operatorname{nin}^{12}{ }^{»}$. L'analyse de comptes rendus d'entrevues d'admission indique que les qualités personnelles exigées pour une bonne candidate en service social s'organisaient autour du modèle traditionnel de la féminité centré sur les vertus oblatives d'accueil, de générosité, de sacrifice, de dévouement, de socialité chaleureuse et de compréhension affective. Une bonne auxiliaire sociale se devait d'être accueillante et d'avoir un contact chaleureux, facile, spontané et ouvert. Ce contact devait révéler «une riche sensibilité», une «stabilité émotive». Un «engagement purement intellectuel» était défini comme suspect, car il renfermait toujours un danger de rationalisation. Les exigences intellectuelles étaient minimisées et on ne s'interrogeait jamais sur le savoir des candidats, sur leur capacité de raisonnement ou de démonstration. On abordait plutôt l'intelligence comme une qualité de la personne reflétant plus des bonnes manières que des aptitudes intellectuelles proprement dites. On insistait davantage sur la compréhension, comme intelligence affective d'autrui, plus près de l'intuition de l'autre, s'exprimant dans un mélange de tact, de sensibilité et d'amour.

Nous lui disons que, en service social, une des qualités des auxiliaires sociales et des personnes qui travaillent à leur formation doit être nécessairement la compréhension. C'est à ce moment que nous pouvons réaliser la belle sensibilité, le respect et l'amour du nécessiteux que possède... ${ }^{13}$

Elle nous raconte quelques-uns de ses cas et elle est pleine d'indulgence, de compréhension, d'acceptation dans toute la

11. La description faite par une candidate est exemplaire de la prégnance de ce sentiment féminin: «Plusieurs motifs m'ont poussé à étudier le service social, tout d'abord, le service social me paraît une carrière essentiellement féminine. Le service social tend à développer chez la femme ce souci du bonheur d'autrui, à renforcer et à orienter ses puissances d'amour et de générosité, enfin le service social familiarise la femme avec les problèmes sociaux et familiaux et ainsi la prépare merveilleusement à sa vocation de maternité [...] En service social, entrent en ligne de jeu non pas seulement les connaissances abstraites mais aussi et surtout les richesses personnelles de la volonté et du coeur.» Le jugement porté par l'évaluatrice se lit comme suit: «maturité plus grande que celle qu'a généralement une jeune fille de 19 ans.»

12. L. Lord, «Avantages du service social catholique», La Presse, 7 décembre 1948.

13. Compte rendu d'évaluation de candidature à l'admission, École de service social, Université de Montréal, 1952. 
force du mot pour ces familles où la maladie lui permet de pénétrer $^{14}$.

Posséder un bon jugement, un désir réel d'aider les gens, le tout exprimé d'une manière très pondérée, très sensée et avec la sensibilité voulue, lorsqu'elle parle de ses visites faites dans les familles nécessiteuses de $X^{15}$.

Ce double niveau de la sociabilité chaleureuse et de la compréhension affective fait partie du profil du candidat mature tout comme le dévouement qui se réalise dans le service aux autres et le respect de l'autorité. Ainsi le don de soi ou le dévouement sont explicitement valorisés et s'actualisent à certains moments dans l'oubli de soi, voire l'abnégation. Ces exigences se formulent, la plupart du temps, à l'intérieur d'un dilemme moral, d'un choix entre «l'égoïsme» et le «don de soi», et une décision en termes d'engagement oblatif est interprétée comme une «motivation mature et sérieuse».

Pour ce qui est du service social, elle y pense depuis longtemps, elle aime le contact avec les gens. Cependant, elle craint de «se donner trop», son «égoïsme» la retenait mais elle a maintenant choisi. Je désire savoir quelles sont exactement ses craintes. Elle sait que si elle s'engage dans un travail comme le service social, elle s'y donnera toute et ça l'effraie un peu. Elle fait la comparaison entre le travail de traductrice qui est purement intellectuel et celui de travailleur social qui engage toute la personne et qui entraîne une grande responsabilité... Sa motivation pour le service social me paraît mature ${ }^{16}$.

La maturité implique aussi une attitude saine face à l'autorité, c'est-à-dire respectueuse, qui doit se réaliser «sans révolte» ni «amertume». Cette docilité se transforme parfois en soumission; elle doit être vécue sur le mode naturel, avec sérénité.

Ne semble pas avoir jamais subi l'autorité familiale même lorsque sa mère malade était exigeante pour elle... ${ }^{17}$ Mlle $\mathrm{Y}$ ne semble pas s'être montrée mécontente, mais a essayé de faire preuve de beaucoup d'amour et de dévouement $[. . .]^{18}$ reconnaît l'échelle des valeurs et le devoir de l'Église d'enseigner et de montrer la route ${ }^{19}$. 
Elle aurait été renvoyée avec deux autres camarades au moment où un nouveau supérieur aurait décidé de rétablir une discipline relâchée. Elle explique que ces trois compagnes se seraient réunies dans une chambre pour causer et leur renvoi aurait servi d'exemple aux autres. Elle raconte les incidents sans révolte et ajoute qu'elle reconnaît la valeur de la formation reçue et n'hésiterait pas à envoyer ses enfants dans ces institutions ${ }^{20}$.

Ce respect de la hiérarchie est en harmonie avec un système de valeurs ascétiques privilégiant le travail, la discipline, l'épargne, l'ordre et la propreté. Cette visée éthique oriente, structure et organise la perception et le jugement porté sur la clientèle. Cette inclination au moralisme où dominent les valeurs d'effort et de sérieux s'actualise même dans le travail de l'auxiliaire sociale, en particulier dans l'enquête et la constitution de ses dossiers.

Habitudes de tenir de bons dossiers a) propres et en ordre; b) dossiers au point; c) rédigés correctement et clairement en choisissant les détails utiles seulement; d) dossiers possédant les documents nécessaires (ou leur copie); e) lettres bien écrites ne dérogeant pas à la tradition établie par le bureau ${ }^{21}$.

Ce système éthique ${ }^{22}$ est en affinité avec la morale catholique ${ }^{23}$ de l'époque et reste aussi lié aux caractéristiques sociales de ces premières générations d'auxiliaires sociales.

Ce service social s'est affirmé comme catholique et s'est intégré au mouvement du catholicisme social dans ce que certains ont nommé la sacralisation du monde profane, c'est-à-dire de catholiciser les principales activités de la vie profane et d'encadrer chaque groupe social dans des activités confessionnelles.

20. Idem, 1950.

21. «Memorandum: consultations de stage», Service familial de Québec, 20 mai 1948.

22. Les problèmes familiaux étaient souvent analysés sur ce registre moral: «Parmi les 24 familles que nous étudierons, nous distinguons deux groupes principaux: a) les familles qui préparent à la société des bons citoyens, b) les autres qui lui donnent des délinquants...» «Tandis que d'autres sont restés dans leur apathie, attendant l'emploi qui ne vient pas et ne comptant que sur les autres pour les faire vivre. Nous croyons plutôt aux chômeurs paresseux qu'aux chômeurs malhonnêtes [...] une famille assistée est-elle négligente ou tout simplement ignorante de l'administration du budget familial...» «Chose assez remarquable, ils ne semblent pas tous conscients de leur malpropreté». Extraits de matériel de thèses de l'époque.

23. «Imaginez le bourgeois formé selon les règles de l'Église. Il se couche et se lève à des heures régulières. Il a ses heures de travail et de repos [...] L'esprit de sa vie est sa régularité [...] Honnête homme, ce bourgeois va tous les dimanches à la messe comme tous les jours de la semaine à son bureau.» B. Groethuysen, Origine de l'esprit bourgeois en France (Paris, N.R.F., 1927), 218. A. Darbel et D. Schnapper, Les agents du système administratif (Paris, C.S.E., Mouton, 1969). 
Ce service social catholique s'opposait au service social neutre ou philanthropique que l'on identifiait chez les protestants. Il s'est différencié de ceux-ci avec ses institutions spécifiques, ses fédérations d'œuvres, ses agences sociales et ses écoles universitaires. Certaines questions ne souffraient d'aucune exception, comme les questions de concubinage, de divorce et de placement d'enfants dans les familles catholiques car toutes ces questions engageaient la confessionnalité. Les directives d'un enseignant en 1946 au sujet d'un cas de concubinage sont des plus explicites: «Les cas de concubinage: Traitement: ou les faire séparer ou les faire marier. Dans les cas où tout le monde croit que la famille est légitime, il est à conseiller dans l'intérêt des enfants de leur conseiller la continence. Dans le cas des personnes libres, il faut les convaincre du mariage et les y préparer au point de vue du sens des responsabilités ${ }^{24} . »$

Cette dimension confessionnelle s'établit à partir de la philosophie thomiste et de la pensée sociale des papes. On a souvent recours aux encycliques de ces derniers pour fonder et légitimer le service social en le situant comme une forme moderne d'apostolat social qui se situe en lien avec le catholicisme social et la hiérarchie ecclésiale.

Forme d'apostolat social catholique, le service social se différentie cependant des pratiques caritatives d'alors dans ce qu'il ne vise pas la sanctification de ses membres et reste critique à l'égard de toute forme de charité et de secours «faite sans discernement». L'enquête est revendiquée comme une nouveauté dans le champ de la philantropie. Une investigation cas par cas devient nécessaire pour déterminer les besoins précis de chacun.

L'aide morale doit toujours primer sur l'aide matérielle: «Le secours direct est préjudiciable à l'individu et à sa personnalité en principe. En pratique, le secours en argent doit être accompagné de conseils pratiques. Afin d'éviter le double emploi dans l'aide procurée à ces écoliers, notre Bureau des œuvres s'est limité à secourir exclusivement ceux dont la demande était justifiée ${ }^{25} . »$

Ces démarches d'enquête constituaient le préalable obligatoire au traitement social ou moral. Car les préoccupations étaient principalement centrées sur le registre familial selon un double objectif: moral et confessionnel, c'est-à-dire relever la famille et la rechristianiser. Ce relèvement de la famille passe par sa remoralisation qui s'exprime dans une représentation traditionnelle de la famille selon une division stricte des rôles entre les sexes.

24. Notes de cours. Assistance sociale générique, École de service social, Montréal, 27 février 1946.

25. A. Lebel, «Le service social à l'école», Relations (septembre 1941); 243. 
La famille est une société complète et le père a autorité dans sa sociétée ${ }^{26}$.

La famille, société hiérarchisée de droit naturel, a pour chef le père. Elle est soumise à des lois morales qu'il faut respecter lorsqu' on veut lui venir en aide ${ }^{27}$. Une des choses essentielles encore dans une famille bien organisée est que ses membres reconnaissent et acceptent les rôles établis. Le père jouant le rôle dominant pour y gagner la vie, la mère dans la tenue de maison; les deux se partageant dans le contrôle des enfants et les décisions à prendre et les enfants ayant un rôle subordonné ${ }^{28}$.

Peu de données existent quant aux caractéristiques sociales des premières générations d'auxiliaires sociales. D'après les données contenues dans les dossiers de l'École de Montréal, plus de soixantequinze pour cent de la population étudiante était féminine durant la décennie 1940. Ce nouveau métier a attiré une population relativement hétérogène. La majorité a une formation secondaire et plusieurs proviennent directement du marché du travail. On retrouve une forte proportion de collets blancs chez les femmes, anciennes infirmières, institutrices, secrétaires et vendeuses, de commis, fonctionnaires et d'ouvriers chez les hommes. Le service social semble avoir constitué pour plusieurs une possibilité de continuer leur action d'apostolat social puisque la majorité était active à l'intérieur de la Jeunesse ouvrière catholique. Pour ceux ou celles dont on possède des données sur l'origine sociale, la majorité provient de classes populaires, le tiers de classes ouvrières, la moitié de classes moyennes (collets blancs et commerçants) et le cinquième de classes supérieures (professionnels). On retrouve une différenciation marquée entre les hommes et les femmes où les hommes sont d'origine plus populaire. Cette différenciation sexuelle se retrouve en outre dans des milieux de pratique où les hommes travaillent dans le champ judiciaire et administratif et les femmes, dans le champ de la famille et de l'enfance.

En résumé, les auxiliaires sociales sont en majorité des femmes provenant de classes moyennes et ouvrières, concevant leur travail en termes de vocation féminine et intervenant dans le champ philanthropique en favorisant le développement d'œuvres sociales non institutionnelles selon un système de valeurs privilégiant la famille, le sens de la hiérarchie, le travail, la discipline et l'ordre.

26. Notes de cours, Philosophie du service social, École de service social, Montréal, 30 novembre 1945 241.

27. E. Bouvier, «Les allocations familiales à OTTAWA», Relations (septembre 1944):

28. T. Morrissette, Conditions de la famille ouvrière à Québec pendant la guerre (Montréal, 1945), 80. 


\section{2 - L'AFFIRMATION PROFESSIONNELLE}

À partir de la fin de la décennie 1950, le savoir professionnel se substitue aux catégories morales et religieuses de description et d'interprétation de la période précédente en s'affirmant comme mode légitime et dominant de détection et d'analyse des problèmes sociaux.

Cette autoreprésentation professionnelle prend son principe de référence autorisé dans la médecine qui constitue l'image réalisée du praticien accompli tant pour le casework que pour l'animation sociale.

Cette intervention (du travailleur social) porte sur des points de tension ou de malaise, de stress comme on dit aujourd'hui, entre l'homme et son milieu social, là où le fonctionnement social bloque, ou menace de s'arrêter, un peu comme l'intervention du médecin porte sur le fonctionnement physique ou mental ${ }^{29}$.

L'animation (sociale) comme l'ancêtre-chirurgien qui découvre laborieusement les techniques chirurgicales [...] une discipline d'intervention à la recherche d'elle-même qui consacre un important effort de réflexion à l'explication de ses intuitions profondes et à l'expérimentation de ses techniques de base ${ }^{30}$.

Le modèle médical et thérapeutique sert de cadre de référence pour identifier les diverses dimensions du métier; on parle explicitement de travail clinique, de diagnostic, de plan de traitement. On insiste sur le caractère professionnel des tâches accomplies qui exigent pour leur réalisation des qualités particulières comme l'équilibre personnel, la capacité d'entrer en relation, l'engagement émotif contrôlé. La relation d'aide remplace ainsi le secours. Le travailleur social se définit comme spécialiste ou expert de la relation dont le rôle est d'agir comme intermédiaire ou facilitateur «pour augmenter la compétence du client au niveau de l'utilisation maximale de ses capacités». «Les mots qu'elle choisit ne font que mal traduire ce qu'elle comprend mieux et j'exprime qu'elle doit vouloir dire que le travailleur social professionnel doit posséder certaines qualifications et compétences que les gens qui consultent ne possèdent pas $^{31} . »$

On passe de la désignation féminine d'auxiliaire sociale à celle masculine de travailleur social, ce qui est parallèle à une déféminisation du service social où la proportion des étudiants masculins passe

29. H. Denault, «L'insertion du service social dans le milieu canadien-français», Service social (avril 1962): 6.

30. M. Blondin, «Vie urbaine et animation sociale», Recherches sociographiques, 9,1-2 (1968): 111.

31. Compte rendu d'évaluation de candidature à l'admission, École de service social, Université de Montréal, 1964. 
du quart à près de la moitié. Les comptes rendus d'évaluation formulent de nouvelles exigences de maturité qui sont en rupture avec les qualités valorisées dans la période précédente.

On se retrouve davantage identifiés aux valeurs de vocation, d'abnégation et de dévouement. Les qualités privilégiées sont désormais l'autonomie, l'initiative et le sens des responsabilités et on insiste sur l'empathie comme capacité de s'identifier à l'autre, de ressentir ce qu'il ressent, dans un engagement émotif contrôlé qui individualise la relation et permet l'expression des sentiments dans une attitude de non-jugement et d'acceptation de l'autre. On se méfie, à cette période, des définitions en termes de vocation et on préfère la conscience sociale à la mission sociale et ce, pour les candidats des deux sexes. «Les différents postes occupés semblent avoir éveillé son sens des responsabilités et son sens social. Elle est intéressée et préoccupée par toutes ces questions. Elle est bien consciente des misères sociales et aime sentir qu'elle travaille à les soulager mais elle ne se donne aucune mission particulière en ce $\operatorname{sens}^{32}$.»

La personnalité du candidat est perçue comme une ressource importante dans la relation d'aide, c'est-à-dire qu'elle fait partie des «techniques» mêmes de travail. Les qualités, comme l'aisance dans la présentation de soi et dans l'interaction sociale et la maîtrise de ses émotions, sont les nouvelles qualités valorisées à cette époque.

Aux qualités interpersonnelles dans l'évaluation d'un bon candidat s'ajoutent les aptitudes proprement intellectuelles qui ne sont plus ramenées à des qualités d'être mais renvoient à des aptitudes à l'analyse, à la démonstration logique. Ces aptitudes sont surtout évaluées à partir de l'analyse de soi permettant de déceler les capacités des candidats à l'insight ou à la saisie «objective» des différentes composantes de leur personnalité, engageant une capacité de mise à distance.

Elle peut analyser, de façon très supérieure, les événements, les situations, elle peut regarder son comportement et celui des autres et en saisir les facteurs et les implications émotionnelles... ${ }^{33}$

Il semble avoir gagné au cours de son expérience une meilleure connaissance de lui-même. Il s'analyse avec assez de franchise et commence à se percevoir en évolution ${ }^{34}$.

Les aptitudes à l'empathie et à l'insight impliquent de nouvelles qualités personnelles qui remplacent le dévouement et le don de soi

32. Idem, 1963.

33. Idem, 1964.

34. Idem, 1962. 
des années 1930 et 1940. Ainsi, on juge maintenant sévèrement ceux ou celles qui manifestent des traits caractéristiques de la période précédente vis-à-vis des figures d'autorité.

Un point plus faible serait celui des relations avec l'autorité. Nous n'avons pas l'impression que A fonctionne parfaitement à l'aise avec les personnes en autorité, elle nous paraît peut-être portée à conserver une relation de dépendance vis-à-vis l'autorité. Elle nous semble avoir perçu toutes les figures d'autorité comme inaccessibles, acceptant difficilement le dialogue. Elle a un peu peur de s'opposer, de forcer la main à l'autorite ${ }^{35}$.

Vis-à-vis l'autorité, elle semble avoir une attitude assez réservée [...] elle n'exprime pas toujours ses opinions principalement avec les personnes en autorités ${ }^{36}$.

Ce nouveau rapport à l'autorité est révélateur des nouvelles attitudes privilégiées et du nouveau système éthique. On exige maintenant une "saine indépendance», une capacité d'être «à l'aise», de «dialoguer avec l'autorité», d'établir des relations de collaboration et d'être capable de défendre son point de vue.

Elle est capable d'initiative et est suffisamment libre de conflits autorité-liberté, dépendance-indépendance pour fonctionner à l'aise avec l'autorité, pour exprimer ses points de vue... ${ }^{37}$

La candidate ne semble pas avoir de grosses difficultés avec l'autorité; dans une situation où elle se trouve dépendante, elle est capable de trouver les moyens pour s'affirmer ${ }^{38}$.

On va même jusqu'à accepter certaines attitudes contestatrices ou revendicatrices. On ne blâme plus le ou la candidate pour de telles attitudes, mais on s'assure de la compétence du surveillant pour faire face à ces comportements. De telles attitudes ne constituent plus des obstacles à l'admission mais plutôt un «défi» pour le milieu de l'enseignement.

Elle a manifesté une agressivité assez bien contrôlée [...] d'autre part on peut soupçonner que cette candidate revendiquera toujours avec beaucoup d'ardeur ses droits et privilèges [...] Cette personne sera un genre de défi pour la surveillante ${ }^{39}$.

En effet l'intelligence très vive, l'enthousiasme et l'incapacité d'accepter passivement ce dont elle est convaincue, éléments que

35. Idem, 1964.

36. Idem, 1965.

37. Idem, 1961

38. Idem, 1963

39. Idem, 1963 
je considère très positifs chez la candidate, contribuent d'autre part à la placer assez souvent dans des situations de conflit avec l'autorité. De façon générale, on peut dire que la candidate n'abdique pas facilement [...] ceci permet de prévoir qu'elle sera probablement une étudiante très exigeante ${ }^{40}$.

L'étude de la relation à l'autorité fait ressortir, tant pour les candidats féminins que masculins, les nouvelles qualités de base qui s'organisent autour de l'initiative, de l'indépendance et du sens des responsabilités. On évalue la maturité des candidats à partir de la capacité de l'individu à mobiliser des ressources pour faire face à des situations difficiles. Tout ce qui peut refléter cette qualité de caractère, comme la détermination dans l'effort, la persévérance devant les difficultés, la maîtrise de soi est valorisé et noté, car cette capacité de mobilisation personnelle reste l'indice premier d'une autodétermination personnelle, qualité primordiale pour l'admission.

Sa vie personnelle, ses activités prouvent qu'il a atteint un certain degré d'autonomie et d'indépendance. Étant donné sa capacité de mobiliser ses énergies devant des frustrations, je recommande ce candidat. Cette expérience laisse présumer que M. a de bonnes ressources intérieures, la capacité de tolérer des situations de «stress» et une faculté d'adaptation plus que la moyenne ${ }^{41}[\ldots]$ réserve quant à la capacité initiale de cette candidate à accepter certaines frustrations inhérentes à la pratique du service social. On sent chez elle un dynamisme nettement agressif en face de la difficulté. Elle ne paraît pas de celles qui acceptent et se soumettent avant d'avoir déployé l'éventail des bons moyens ${ }^{42}$.

Le discours professionnel des travailleurs sociaux se présente comme une rupture dans le questionnement de la conception religieuse, résiduelle ou morale de la pauvreté comme fautes, incapacités ou carences personnelles et dans la construction d'une nouvelle définition psychosociale, c'est-à-dire comme produit de mécanismes sociaux, impliquant le passage d'une attitude ou action répressive et philanthropique à une attitude ou action persuasive et thérapeutique. Cette reformulation et la mise en circulation de nouvelles catégories de perception, de désignation, d'interprétation de la famille et de la pauvreté dans le renversement des images et des attitudes traditionnelles participent du processus plus large de professionnalisation du champ de l'action sociale. Le travailleur social, en s'insérant dans le

40. Idem, 1962.

41. Idem, 1965.

42. Idem, 1963. 
champ philanthropique, essaie d'en modifier la structure de façon substantielle. Il est amené à adopter une position de contestation de la vision traditionnelle du travail philanthropique. Il s'oppose tant à la pratique bénévole des gens d'œuvres, leur reprochant leur amateurisme et leur moralisme, qu'à la pratique de la charité institutionnelle des religieuses ${ }^{43}$, critiquant leur autoritarisme et leur position quasi monopolistique dans le champ de la philanthropie. Celui-ci met de l'avant des modes d'intervention qui valorisent, contrairement aux gens d'œuvres, la continuité, la qualification, les méthodes scientifiques ou la rationalisation des pratiques d'aide et, en opposition aux religieuses, la désinstitutionnalisation des populations prises en charge. Ceci se traduit par le développement d'agences familiales avec du personnel rémunéré et spécialisé correspondant à une expansion du marché en service social. Les services se développent, et les agences sociales se consolident et se multiplient dans ce que certains ont nommé la «provincialisation des services sociaux francophones ${ }^{44}$ ». Le nouveau champ se constitue et se structure sous le contrôle des travailleurs sociaux professionnels à travers le regroupement professionnel dans la Corporation des travailleurs sociaux professionnels et institutionnels dans la Fédération des services sociaux à la famille, ce qui traduit la reconnaissance sociale et juridique du service social et le contrôle effectif des travailleurs sociaux dans le champ des services sociaux. Cet élargissement des services sociaux correspond au développement de l'État dans le champ social. Ce dernier favorisait et soutenait le développement d'une main-d'œuvre professionnelle dans le champ de l'action sociale. Aussi l'autoreprésentation professionnelle des travailleurs sociaux était partagée, à l'époque, par les commissions d'enquête gouvernementale dont la Commission Parent sur l'éducation en 1964 et la Commission Boucher sur l'assistance publique en 1963. Chacune reconnaissait au service social une fonction professionnelle de prévention et de réhabilitation en recommandant un financement direct des agences selon leurs programmes et non plus au moyen de la gestion de l'assistance publique.

Il s'agit en définitive pour le ministère d'être autre chose qu'une vaste machine distributrice [...] Dès lors apparaît la nécessité absolue d'un personnel compétent d'autant plus que les indigents sont des êtres humains souvent aux prises avec des problèmes extrêmement délicats. Le Comité croit essentiel l'engagement

43. Un des débats principaux concerne le placement des enfants dans les foyers nourriciers plutôt qu'à l'orphelinat.

44. G. Renaud, L'éclatement de la profession en service social (Montréal, Éditions SaintMartin, 1978). 
d'un personnel déjà formé aux disciplines du bien-être social [...] Le ministère a un besoin pressant de gradués d'université dans les domaines social... ${ }^{45}$

La participation du travailleur social est basée sur sa connaissance du comportement humain avec ses facteurs sociaux, sur sa compétence [...] Tout d'abord, il faut reconnaître au service social le statut de service fonctionnel [...] Le service social remplit ses fonctions propres dans un rapport collatéral de coopération et de consultation. Nous recommandons qu'un service social scolaire soit mis à la disposition de toutes les écoles élémentaires et secondaires, dans la mesure où l'on bénéficiera du personnel qualifié requis ${ }^{46}$.

Cette professionnalisation du service social par la certification universitaire de certains de ces agents a favorisé l'accroissement de la division du travail entre les travailleurs sociaux de formation universitaire et les conseillers sociaux de formation collégiale, c'est-à-dire que certaines tâches touchant à la gestion, la conceptualisation, l'évaluation et la supervision ont été monopolisées par les travailleurs sociaux professionnels, laissant aux conseillers sociaux les tâches et les secteurs d'action les moins prestigieux et impliquant une plus grande part de travail administratif ${ }^{47}$.

La division sexuelle du travail s'est accentuée à cette période. À la division horizontale du travail entre les sexes selon les champs de pratique de la période précédente s'ajoute et se substitue une division verticale du travail entre les sexes où la gestion et l'organisation des services sociaux deviennent plus le fait de travailleurs sociaux masculins et la pratique directe, de travailleurs sociaux féminins, ce qui n'a pas été sans effet sur une redéfinition thérapeutique de la pratique au niveau de l'enfance, de la famille et de la santé et une revendication du service social comme service public dans le champ du bien-être.

Cette professionnalisation du service social amène une modification, une substitution de la population quant au sexe et à l'origine sociale. Comme nous l'avons noté précédemment, la population étudiante se masculinise, atteignant plus de $40 \%$ durant cette période. On se retrouve en outre devant une population ayant une origine sociale légèrement plus élevée que durant la période précédente.

45. Rapport du comité d'étude sur l'assistance publique (Québec, Gouvernement du Québec, 1963), 146-147.

46. Rapport de la Commission royale d'enquête sur l'enseignement dans la province de Québec, 3: 328-329 et 376.

47. La maturité est invoquée pour marquer les différences entre les travailleurs sociaux professionnels et les conseillers sociaux. Elle renvoie au thème de l'équilibre personnel, la capacité d'entrer en relation, l'engagement émotif contrôlé, c'est-à-dire la maîtrise de soi. 
Tableau 1

Distribution de la population étudiante en service social à l'Université de Montréal selon l'occupation du père (1964-1966)

\begin{tabular}{|l|c|c|}
\hline & $\begin{array}{c}\text { Hommes (40\%) } \\
\%\end{array}$ & $\begin{array}{c}\text { Femmes (60\%) } \\
\%\end{array}$ \\
\hline Haute administration et industriels & 2,5 & 0 \\
\hline Professionnels & 5,0 & 27,5 \\
\hline Total & 7,5 & 27,5 \\
\hline $\begin{array}{l}\text { Cadres du public, enseignants, } \\
\text { semi-professionnels }\end{array}$ & 7,5 & 17,5 \\
\hline Cadres du privé & 5,0 & 12,5 \\
\hline Commerçants & 20,0 & 7,5 \\
\hline Cols blancs & 10,0 & 12,5 \\
\hline Total & 42,5 & 50,0 \\
\hline Contremaîtres & 5,0 & 5,0 \\
\hline Ouvriers qualifiés & 7,5 & 2,5 \\
\hline Ouvriers semi-qualifiés et manœuvres & 22,5 & 5,0 \\
\hline Total & 35,0 & 12,5 \\
\hline Agriculteurs & 15,0 & 10,0 \\
\hline
\end{tabular}

Source: $\quad$ Compilation faite à partir des dossiers étudiants, École de Service social, Université de Montréal.

Comme l'indique le tableau 1, on se retrouve devant une forte surreprésentation des classes moyennes. Cette surreprésentation s'établit pour les deux sexes bien que l'on retrouve une variation importante de l'origine sociale selon le sexe. Les femmes proviennent majoritairement de classes moyennes et supérieures et les hommes de classes ouvrières et moyennes ${ }^{48}$. Les hommes sont surtout des fils d'ouvriers et de commerçants et les femmes, filles de professionnels et de semiprofessionnels ${ }^{49}$.

48. Ces variations proviennent, pour une part, de la modification des conditions d'entrée à la formation qui passe des exigences du secondaire à celles du baccalauréat. Cette modification des «propriétés scolaires des occupants» a favorisé le renouvellement/substitution des agents par rapport à l'époque antérieure.

49. On retrouve le même phénomène en France et aux États-Unis. Muel-Dreyfus, «L'initiative privée: le terrain de l'éducation spécialisée», Actes de la recherche en sciences sociales (avril-juin 1980): 51-64. G. L. Gockel, «Silk Stockings and Blue Collars» (Chicago, National Opinion Research Center, University of Chicago, 1966). 
Tableau 2

Distribution de la population étudiante universitaire selon l'occupation du père (universités francophones, 1961) ${ }^{1}$

\begin{tabular}{|l|c|c|}
\hline & Hommes & Femmes \\
& $\%$ & $\%$ \\
\hline Professionnels & 8 & 22 \\
Salariés administration & 6 & 2 \\
\hline Semi-professionnels & 2 & 24 \\
Petite administration, commerce & 26 & 8 \\
Cols blancs & 10 & 12 \\
\hline Ouvriers spécialisés & 21 & 4 \\
Ouvriers semi-spécialisés & 6 & 3 \\
Manœuvres & 8 & 6 \\
\hline Cultivateurs & 11 & 7 \\
\hline Indéterminés & 3 & \\
\hline
\end{tabular}

1 J. Brazeau et al., Les résultats d'une enquête des étudiants dans les universités de langue française au Québec (Montréal, Département de sociologie, Université de Montréal, 1962), Appendice D, tableau 98.

Comparativement à la population universitaire (tableau 2), les étudiantes en service social sont légèrement surreprésentées au niveau des classes supérieures et moyennes (cadres publics, semi-professionnels et cols blancs) et sous-représentées au niveau des classes ouvrières. Quant aux étudiants masculins en service social, leur profil est davantage similaire à ceux des étudiants universitaires en général avec une légère sous-représentation des classes supérieures (professionnels) et une légère surreprésentation des classes moyennes (cadres publics, enseignants, semi-professionnels).

Comparativement à la distribution de la population en général, les femmes en service social montrent une sous-représentation des classes populaires, surtout ouvrières, une surreprésentation des classes supérieures et une forte surreprésentation des classes moyennes. Quant aux hommes en service social, on note une sous-représentation des classes supérieures, une légère sous-représentation des classes ouvrières et une surreprésentation des classes moyennes.

Tout permet de supposer que l'extension du marché des services sociaux, la constitution d'un champ professionnel lié à la reconnaissance juridique, sociale et universitaire du métier ont attiré une nouvelle population, qui a favorisé une redéfinition technique et 
sociale du métier dans l'affirmation de nouvelles dispositions éthiques en rupture par rapport au système d'éthique de la période précédente ${ }^{50}$. On ne retrouve plus l'indignation morale, le sens de la hiérarchie et l'ascétisme de la période précédente; on favorise maintenant une plus grande liberté concernant les rôles prescrits, en particulier à l'intérieur de la famille où l'on valorise une égalisation des rôles. Pour ce qui est du métier, on insiste sur des attitudes de neutralité, de distance au rôle, d'autonomie vis-à-vis de l'autorité et du système institutionnel.

En résumé, cette période voit s'affirmer une redéfinition professionnelle du service social concomitant à un renouvellement du système de valeurs parallèle à une déféminisation et une modification des effectifs avec une origine sociale plus élevée par rapport à la période précédente.

\section{3 - LA REMISE EN QUESTION PROFESSIONNELLE}

La décennie 1970 voit apparaître une contestation de cette identité professionnelle par le pouvoir d'État, les nouvelles professions sociales et les groupes volontaires d'usagers. Cette contestation s'établit en outre dans un contexte de bureaucratisation et de technocratisation des services sociaux qui amènent à une déqualification relative du service social.

Sans entrer dans l'ensemble systématique des transformations qu'a connues le système de bien-être québécois depuis la fin des années 1960, soulignons le processus accéléré de sécularisation, de rationalisation des divers appareils de bien-être qui s'est traduit par une croissance et une diversification des effectifs et par une concentration-centralisation des processus de décision, de planification et d'évaluation, entraînant une complexification du système interne des relations professionnelles et institutionnelles. Cette croissance et cette emprise institutionnelle et bureaucratique sur les pratiques d'intervention entraînent une restructuration et une diversification du champ professionnel dont un des effets demeure, dans bien des cas, la substitution à l'ancienne solidarité professionnelle ou corporative, une solidarité plus syndicale, fondée sur une complémentarité des intérêts interoccupationnels.

50. Si la clientèle était perçue et définie à partir de critères simples de moralité à l'époque antérieure, elle est maintenant redéfinie, à l'époque professionnelle, à partir de critères thérapeutiques ou «scientifines» qui se structurent souvent autour de l'opposition: traditionnelle/ moderne. La clientèle est jugée comme traditionnelle dans ses comportements et ses attitudes, ce qui expliquerait ses problèmes, et le travailleur social serait du côté moderne permettant, par la relation d'aide ou l'action sociale, de favoriser son développement, sa modernisation. 
Depuis le début des années 1970, le champ des services sociaux a connu des transformations importantes à la suite des travaux de la Commission d'enquête sur la santé et les services sociaux (Commission Castonguay-Nepveu) qui avait été mise sur pied en 1966 et dont les travaux durèrent plus de six années. Ainsi en décembre 1970 fut créé le ministère des Affaires sociales à partir de la fusion des anciens ministères de la Santé, de la Famille et du Bien-être social, ce qui implique une redéfinition de leur vocation respective. Puis en 1971 fut adoptée à l'Assemblée nationale la Loi sur les services de santé et les services sociaux (L.Q. 1971 C. 48), loi qui provoqua des bouleversements considérables tant sur les plans de la conception que de l'organisation et la distribution des services sociosanitaires, comme le note avec justesse Renaud à ce propos:

En somme, un chambardement d'envergure prit place. On supprima certains organismes. D'autres furent créés. D'autres virent leurs rôles et fonctions redéfinis. Plusieurs procédures de gestion furent modifiées et des responsabilités de gestion furent attribuées à des personnes autrefois totalement étrangères à ce travail. Et finalement, la structure d'ensemble fut clarifiée sur papier, en distinguant ce qui était tâches d'exécution (le «réseau») et tâches de conception et en reclassant l'ensemble des organisations du réseau en quelques catégories bien définies ${ }^{51}$.

$\mathrm{Au}$ plan des services sociaux, les quarante-trois agences et les six centres psychosociaux furent transformés en quatorze centres de services sociaux à partir de 1973. Cette réorganisation amena quatre changements majeurs: premièrement, l'apparition d'une gestion spécialisée; deuxièmement, la normalisation et la standardisation des actes et des tâches; troisièmement, l'introduction d'autres professionnels que les travailleurs sociaux; et quatrièmement, la syndicalisation de ces professionnels.

Dans ce contexte, de nouveaux groupes d'agents de formation universitaire et collégiale sont amenés à contester la position quasi monopoliste des travailleurs sociaux dans le champ des services sociaux en revendiquant l'accès aux postes. Ceci s'est traduit par la création institutionnelle d'un nouveau poste, celui d' «agent de relations humaines ${ }^{52}$ » qui possède une description de tâches identique à

51. M. Renaud, «Réforme ou illusion? Une analyse des interventions de l'État québécois dans le domaine de la santé», Sociologies et Sociétés, 9,1 (avril 1977): 138.

52. Ces redéfinitions institutionnelles du poste sont vécues comme une remise en question du service social: «Escamotage de la spécificité des professionnels par les agents de relations humaines [...] La définition du travailleur social devient vidée de toute spécificité étant identique à celle de l'agent de relations humaines». M. Rinfret et R. Ziniga, Le stage en service social (Montréal, 1982), 38. 
celle du poste de travailleur social, mais dont l'occupation n'exige plus le titre de travailleur social. La contestation ne se limite pas au poste, mais touche aussi la validité du savoir professionnel qui est critiqué en particulier par les gestionnaires pour ses effets «contreproductifs» sur la clientèle. L'action des services sociaux est de plus située dans le cadre plus général de l'État et du développement social où l'établissement du service social est défini comme un «système dans lequel le praticien n'est qu'un des éléments ${ }^{53}{ }$.

Les règles du jeu sont différentes; le client ne s'adresse plus à un praticien d'abord, il s'adresse à un établissement qu'il tiendra responsable de lui rendre des services et lorsque ce service sera rendu, qu'il tiendra responsable de la qualité du service rendu. Il demandera de l'établissement, qu'il lui offre un personnel ${ }^{54}$.

Ce déplacement du lieu de responsabilité et d'autorité du professionnel à l'établissement a amené les agents du service social à intervenir davantage en fonction des législations sociales et a, de ce fait, modifié leur mandat d'intervention. La mise en place de nouveaux cadres législatifs, en particulier dans le domaine de la protection sociale et de la prévention sociosanitaire, a favorisé la mise en place de deux modèles d'intervention: un modèle sociojuridique et un modèle sociomédical. Cette dualisation des systèmes d'aide est vécue par les travailleurs sociaux sur le mode du contrôle étatique pour «judiciariser et médicaliser la pratique sociale ${ }^{55}$ ».

Ces transformations institutionnelles s'inscrivent dans la double exigence de rationalisation et de rentabilisation des services publics, c'est-à-dire de régulation et de contrôle des postes, des ressources et du budget. Concernant l'augmentation des coûts des services publics et la volonté politique «de ralentir la croissance des dépenses sociales, en particulier en éducation et dans les affaires sociales ${ }^{56} »$, le projet de

53. J.-P. Duplantie, «Être un établissement responsable», Le travailleur social, 45,3 (automne 1977): 115.

54. Idem, 116-117.

55. «La protection de la jeunesse», Intervention, 52 (1978). L'institutionnalisation ou la technocratisation des services sociaux a produit une stratification et une différenciation entre groupes de travailleurs sociaux. La perspective professionnelle continue d'être véhiculée, en particulier par les cadres intermédiaires, chefs de services, ceux ayant un rôle de supervision ou d'encadrement professionnel. En position intermédiaire dans le système de stratification, ils véhiculent une orientation professionnelle qui fonde leur revendication d'autonomie vis-à-vis de leur supérieur hiérarchique et légitime leur autorité de fonction face à leurs subalternes. Mais cette orientation est peu reprise par les travailleurs sociaux praticiens plus jeunes qui vivent un rapport plus désenchanté quant à leur pratique, subissant les contraintes de l'encadrement bureaucratique et professionnel et dont les perspectives de mobilité individuelle par la voie professionnelle sont réduites par une bureaucratisation des activités.

56. D. Bouchard et P. Sénéchal, «L'évolution des coûts des services sociaux au Québec», Service social, 27,1: 64-65. 
déprofessionnaliser les pratiques d'intervention apparaît, pour plusieurs, comme l'affirmation d'une logique technocratique dans le champ des services sociaux.

En résumé, ces transformations institutionnelles agissent sur la définition du service social par les modifications objectives des conditions de travail, des conditions d'exercice des pratiques d'intervention, bref, de la division sociale du travail dans les «industries» de bienêtre, ce qui crée une situation de crise larvée qui s'exprime dans des formes multiples de relégation et de bureaucratisation des activités occupationnelles, situation favorisant ou obligeant à une rupture ou à une contestation du modèle professionnel qui apparaît en porte-à-faux quant aux transformations dans le champ du bien-être.

\section{4 - UNE REDÉFINITION FÉMINISTE}

Ce processus de technocratisation des services sociaux permet de comprendre les essais de redéfinition du service social qui sont apparus dans le champ des services sociaux à la fin de la décennie 1970. L'une de ces définitions s'est affirmée comme critique, féministe et interventionniste. Cette nouvelle identité est aussi liée à la promotion d'un nouveau système de valeurs qui s'établit dans un contexte de reféminisation des agents du service social et une modification du recrutement quant à l'origine sociale.

Cette redéfinition se nourrit d'une critique féministe de la technocratisation et de la professionnalisation des pratiques sociales. L'intervention de l'État n'est plus pensée en termes de développement social mais plutôt en termes de contrôle social et de victimisation des intervenantes sociales et des clientèles des services sociaux. L'ensemble des mécanismes institutionnels et professionnels à l'intérieur des services sociaux sont jugés discriminatoires à cause des définitions implicites de la femme qui y sont véhiculées, définitions qualifiées de «préjugés sexistes». La démarche thérapeutique est maintenant rejetée dans ce qu'elle vise à «ajuster, adapter, rééduquer aux valeurs dominantes et à réinsérer les femmes dans leurs rôles sociaux stéréotypés ${ }^{57} \gg$. On se définit comme intervenante plutôt que thérapeute pour se démarquer de toute référence psychologique ou médicale. Le modèle médical et professionnel qui fonctionnait comme modèle de référence à la période précédente est maintenant perçu et défini comme mécanisme d' «infantilisation» des femmes. La dépendance au système médical est critiquée tout comme la relation thérapeutique,

57. J. Labrosse, L'intervention féministe en service social (Montréal), 3. 
réinterprétée en termes de rapport de «domination et de subordination de la femme à son thérapeute ${ }^{58}$ ».

La thérapie est sexiste, on oriente les femmes en fonction [...] des stéréotypes, une femme normale. C'est une femme qui correspond à des stéréotypes, une femme normale [...] soumise, douce, gentille [...] donc ça aide pas finalement au développement des femmes; tout ce que ça aide c'est de les entrer dans un cadre social donné où les femmes sont opprimées ${ }^{59}$.

Maintenant plus ça va, plus on se rend compte qu'on est toujours intervenu auprès des femmes de façon très sexiste [...] les intervenants pourront pas être aussi chauvins qu'ils l'étaient, parce qu'ils vont se sentir mal dans leurs bottines ${ }^{60}$.

Cette remise en question des diverses formes d'aide professionnelle s'accompagne d'un renouvellement de l'analyse des problèmes de la clientèle des services sociaux. On souligne le caractère féminin de la clientèle en insistant sur le vécu spécifique des femmes clientes. Le thème du sexisme devient la notion charnière de ce nouveau discours, car le vécu des femmes est interprété en termes d'oppression qui provient de l'enfermement des femmes dans des rôles traditionnels qui, selon les termes mêmes des intervenantes, «confinent les femmes dans les rôles de servitude, limitatifs et contraignants». La définition de la féminité qui était valorisée durant la première période est maintenant réinterprétée comme oppression. Malgré des divergences sur l'explication de l'oppression spécifique des femmes qui, pour certaines, provient du processus de socialisation, pour d'autres, de la société patriarcale et capitaliste, toutes s'entendent à dénoncer les rôles et les tâches socialement attribuées aux femmes. Certains rôles comme ceux d'épouse, de mère et de ménagère sont l'objet des plus virulentes critiques. C'est certes celui de la mère qui est le plus remis en question; on tourne en dérision la «job» de mère, car elle représente le prototype du rôle qui «se définit en fonction des autres».

Les rôles sociaux assignés aux femmes sont différents de ceux attribués aux hommes et que ceux-ci sont limitatifs et aliénants pour les femmes, avec les effets négatifs suivants: culpabilité, perte d'estime de soi, passivité, dépendance, dépression, etc. ${ }^{61}$

58. Idem, 4 .

59. Entrevues, A. D., novembre 1984.

60. Entrevues, D. L., décembre 1984.

61. Nous femmes ou une expérience d'intervention féministe (Montréal, CSSMM, 1982), 4. 
Quand je suis avec des femmes de 58 ans [...] qui se retrouvent devant une détresse tellement profonde, de se rendre compte que toute leur vie a passé à être au service du mari et des enfants, et que finalement, une fois que ces gens-là se sont ressourcés et volent de leurs ailes, elles sont mises aux rebuts, au rencart [...] Pour répondre à des rôles qu'elles n'ont pas choisis ${ }^{62}$.

Je trouve ça tellement injuste que les femmes aient ce prix-là à payer pour être mère, pour répondre à des rôles qu'elles n'ont pas choisis ${ }^{63}$.

Je me sentais libérée de ce rôle de mère qui exige le don total de soi $^{64}$.

La question féminine remplace la question familiale des décennies précédentes ou, plutôt, l'attention à la condition féminine amène à réintroduire dans le champ des services sociaux des préoccupations et des attitudes nouvelles vis-à-vis de la famille. On reformule, on redécouvre des problèmes nouveaux à l'intérieur de la famille en réintroduisant la notion de pouvoir dans la dynamique familiale. La question de la violence familiale ou conjugale est exemplaire de cette reformulation qui construit un nouveau langage autour de la femme battue qui devient objet de visibilité sociale dans le développement d'outils de dépistage de l'homme abusif, de la femme violentée et de l'escalade ou cycle de la violence conjugale et familiale et des modes d'intervention pour «travailler le vécu de crise», pour «ouvrir et soutenir le vécu de violence», et pour favoriser le processus de «conscientisation et de libération ${ }^{65}$ ». D'autres problèmes comme ceux des femmes chefs de famille monoparentale, que l'on nommait fillesmères à la première période et mères-célibataires à la seconde, sont réinterprétés dans le cadre de l'analyse de l'oppression spécifique des femmes. Celles-ci ne sont plus définies comme malades ou dépressives mais plutôt comme victimes d'un «mal de vivre», objet de la solidarité de l'intervenante féministe ${ }^{66}$.

Les femmes des classes populaires constituent une «populationcible» privilégiée pour l'intervention, car elles sont jugées comme vivant plus que d'autres ce conditionnement social à des rôles traditionnels qualifiés de «stéréotypés et de sexistes».

62. Entrevues, L. M., octobre 1984.

63. Entrevues, P. C., janvier 1985.

64. T. Longpré, «La foulée des femmes», Possibles (1985).

65. G. Larouche, Guide d'intervention auprès des femmes violentées (Montréal, C.T.S.Q., 1985), 46.

66. P. Gingras, Une pratique féministe (Québec, 1984), 7. 
C'est surtout dans les classes populaires où les stéréotypes sont encore beaucoup plus rigides, plus forts où les hommes sont encore beaucoup plus [...] beaucoup plus hommes et les femmes sont encore beaucoup plus femmes, tu sais, je pense que c'est encore plus rigide ${ }^{67}$.

Les femmes à faible revenu sont, en général, des femmes qui possèdent des valeurs familiales traditionnelles, sortent difficilement de leur milieu et possèdent un «gros bon sens» et sont fondamentalement conservatrices [...] elles sont en outre, de grandes consommatrices de télévision, de rêves et de scandales [...] elles sont un peu crédules...

Ces femmes s'associent à une vision stéréotypée de leur rôle comme bonne mère, bonne amante, enfin, comme le prototype féminin ${ }^{68}$.

Ce jugement commande l'urgence d'intervenir. C'est pourquoi il ne faut pas se surprendre de l'utilisation de la confrontation pour lutter contre ces «préjugés sexistes».

Il ne faut pas éviter de confronter la femme battue. Une attitude de surprotection nuit aux femmes violentées. Ces dernières, comme tous les êtres humains, se cachent certaines réalités, et elles peuvent y être confrontées. Elles peuvent progresser en prenant conscience de leurs contradictions. Ex.: «Tu restes parce que tu crains de ne pas être capable d'éduquer seule les enfants. Par contre, ton mari travaille tous les jours et il est en état d'ébriété cinq soirs par semaine. C'est donc toi qui de toute façon élèves seule tes enfants [...] En réalité, est-ce que c'est la solitude qui te fait peur $^{69}$ ?

L'intervention n'obéit plus aux règles de la pratique professionnelle faites d'objectivité, de neutralité éthique et de distance au rôle. La situation d'oppression des femmes nécessite une action qui engage l'intervenante à partager son vécu de femme, à établir «un esprit de connivence et de solidarité» et à prendre fait et cause pour la «libération» de la femme.

Divulguer son orientation quant à l'analyse de la situation des femmes dans la société [...] s'impliquer personnellement comme intervenante ${ }^{70}$.

67. Entrevues, A. D., novembre 1984.

68. H. Lechasseur et G. Martin-Beausoleil, «Les femmes et les inégalités socioéconomiques: La Gicogne, maison des femmes de Matane», Service social, 32,3 (1983): 404.

69. G. Larouche, op. cit., 47.

70. Entrevues, E. L., décembre 1984. 
Implication personnelle de l'intervenante à travers l'expression et le partage de sentiments, de situations, d'expériences et de difficultés sur les sujets abordés ${ }^{71}$.

Dans ce contexte, l'intervention se base sur une conscientisation féministe qui se présente comme une compétence idéologique de compréhension et d'analyse critique de la condition féminine. Mais cette compétence féministe n'est réductible ni à un militantisme ni à un savoir-faire technique. La banalisation possible de l'intervention en termes de technicisation par l'État est vécue comme une menace toujours possible de récupération ou de contrôle et exige vigilance et attention.

[...] risque de devenir un autre «truc», une autre technique dans la vaste panoplie des «kits» maintenant disponibles sur le marché de la psychothérapie.

Ça peut devenir un kit pour travailler avec les femmes. Il suffirait d'une technicienne à toute fin pratique pour les appliquer. Ce sera une façon de gérer les femmes, c'est une récupération. On en fait des kits puis je ne suis pas certaine non plus que ça va déboucher vers une action sociale de changement ${ }^{72}$.

L'intervention féministe vise à «modifier les attitudes et les comportements ${ }^{73}{ }^{»}$ selon un système de valeurs différent des périodes précédentes. Si à la première période on pouvait identifier une éthique ascétique s'organisant autour des valeurs de l'épargne, de l'effort et du travail, on peut qualifier la nouvelle éthique de cette période d' «expressive» du fait qu'elle valorise l'expression de soi, de son vécu, de ses émotions, de ses fantaisies, de sa tristesse, de sa colère, bref, de ses besoins et de ses désirs comme individu et comme femme. Cette affirmation de soi exige d'être «bien dans sa peau» et dans ses rapports avec autrui. Une attention particulière est donnée au corps comme lieu de sensation et de plaisir. On revendique le droit au plaisir et à la réappropriation de son corps, de sa sensualité et de sa sexualité.

Cette morale garde des connotations hédonistes dans l'insistance sur l'individualisme ${ }^{74}$ et le plaisir et anti-autoritaires dans la critique

71. Entrevues, P. M., novembre 1984.

72. Entrevues, L. M., octobre 1984.

73. C. Corbeil et al., L'intervention féministe (Montréal, Éditions Saint-Martin, 1983).

74. Cette morale rejoint ce que Bell nomme «l'idée de moi antinomiste»: «Le jugement moral vient de l'individu et non d'une institution; le discernement vient de l'expérience et non de la tradition. L'idée d' «identité authentique» constitue la norme à laquelle les individus doivent obéir: sous le joug des institutions et des organisations qui lui imposent des «rôles», «l'homme = tout» est dépersonnalisé et détruit. Alors que l'idéal du XIX ${ }^{\mathrm{e}}$ siècle mettait l'accent sur la liberté, sur le désir d'être libre de toute obligation imposée, l'idéal du $\mathrm{XX}^{\mathrm{e}}$ siècle met l'accent sur la «libération», sur l'absence de toute contrainte institutionnelle». D. Bell, «La nouvelle classe, un concept bâtard», Revue française de sociologie (oct.-déc. 1983): 715. 
des diverses formes de domination familiale, professionnelle et institutionnelle. Elle privilégie fortement l'autonomie ${ }^{75}$ comme capacité $^{\prime}$ d'identifier, d'affirmer et de négocier ses besoins et valorise l'égalité et la démocratisation des relations dans le couple qui se réalisent par une indifférenciation des rôles et des tâches à l'intérieur du couple. Ce parti pris pour l'expression et le partage du vécu, pour la réappropriation de son corps et de sa sexualité, pour l'affirmation et l'actualisation de soi et la capacité de négocier ses besoins avec son environnement fonctionne comme nouveau modèle de vie personnelle et familiale explicitement affirmé et diffusé auprès de la clientèle des services sociaux.

Se voir en tant qu'individu permet à la femme battue de remettre en question la cellule familiale et d'orienter ses choix en termes de rupture ou de renégociation du contrat de mariage [...] Se reconnaître le droit d'avoir des besoins représente un pas important pour se centrer sur soi. L'intervenante doit dans bien des cas persuader la femme battue que tout individu a des besoins. Ex.: «Tous les êtres humains ont des besoins d'amour, de réalisation personnelle, d'écoute, etc. On peut ne pas se les avouer mais c'est impossible de ne pas en avoir.»

Encore une fois, les tâches seront employées afin de permettre aux femmes de redécouvrir leur corps: se regarder dans le miroir et apprendre à apprécier son corps (sans référer aux standards publicitaires), le caresser et se procurer du plaisir afin de bien connaître ses besoins et de s'autoriser à une sexualité basée sur ses besoins ${ }^{76}$.

Ce renouvellement du langage et des pratiques est parallèle à une reféminisation et une démocratisation plus forte du recrutement relativement à l'origine sociale. On retrouve aussi au début des années 1980 un profil de la population étudiante radicalement différent de celui de la décennie 1960.

La déféminisation que l'on notait dans la décennie 1960 est maintenant inversée puisque la population étudiante est de plus des trois quarts comme au début des années 1940. Malgré le faible nombre d'étudiants masculins, on retrouve peu de différence quant à l'origine sociale entre les populations féminine et masculine en service social. Elles se présentent comme beaucoup plus homogènes entre elles comparativement à celles des décennies précédentes comme l'indique le tableau 3.

75. Le thème: autonomie, autogestion, autodéveloppement, auto-organisation constitue une dimension importante de cette «éthique de la libération».

76. G. Larouche, op. cit., 48. 
Tableau 3

Distribution de la population étudiante en service social à l'Université de Montréal selon l'occupation du père (1980-1982)

\begin{tabular}{|l|c|c|}
\hline & $\begin{array}{c}\text { Hommes (15\%) } \\
\mathbf{\%}\end{array}$ & $\begin{array}{c}\text { Femmes (85\%) } \\
\mathbf{\%}\end{array}$ \\
\hline Haute administration et industriels & 0 & 1,4 \\
\hline Professionnels & 10,0 & 11,3 \\
\hline Total & 10,0 & 12,7 \\
\hline $\begin{array}{l}\text { Cadres du public, enseignants, } \\
\text { semi-professionnels }\end{array}$ & 10,0 & 11,3 \\
\hline Cadres du privé & 5,0 & 4,9 \\
\hline Commerçants & 0 & 9,2 \\
\hline Cols blancs & 30,0 & 17,7 \\
\hline Total & 45,0 & 43,2 \\
\hline Contremaîtres & 10,0 & 11,3 \\
\hline Ouvriers qualifiés & 20,0 & 13,4 \\
\hline Ouvriers semi-qualifiés et manœuvres & 15,0 & 17,0 \\
\hline Total & 45,0 & 41,8 \\
\hline Agriculteurs & 0 & 2,1 \\
\hline
\end{tabular}

Source: $\quad$ Compilation faite à partir d'une enquête auprès de la population étudiante en service social en 1980, 1981 et 1982.

Le modèle de recrutement des femmes en service social a fortement varié depuis les années 1960 jusqu'à rejoindre celui des hommes. Ainsi les étudiantes en service social de l'Université de Montréal proviennent majoritairement des classes populaires (cols blancs: $17 \%$, classe ouvrière: $41,8 \%$, total $58,8 \%$ ). Elles se distinguent encore légèrement des hommes dans la mesure où une proportion légèrement supérieure d'étudiants sont issus des classes populaires (cols blancs: $30 \%$ et classe ouvrière: $45 \%$, total: $75 \%$ ). Malgré un recrutement plus populaire que la décennie 1960, on trouve une surreprésentation des classes moyennes tant chez les hommes que chez les femmes, comme durant la décennie 1960, mais une plus forte provenance de faction inférieure (collets blancs) des classes moyennes durant la décennie 1980.

Si l'on compare cette population étudiante en service social (tableau 4) avec la population étudiante universitaire, on note une légère sous-représentation des professionnels et une forte sousreprésentation des cadres du privé tant chez les hommes que chez les femmes en service social; une forte surreprésentation des ouvriers ( $14 \%$ de plus pour les hommes et $19 \%$ de plus pour les femmes); une 
Tableau 4

Distribution de la population universitaire selon l'occupation du père

\begin{tabular}{|l|c|c|}
\hline & $\begin{array}{c}\text { Hommes } \\
\%\end{array}$ & $\begin{array}{c}\text { Femmes } \\
\%\end{array}$ \\
\hline Professionnels & 12 & 14 \\
\hline Haute administration & 3 & 4 \\
\hline $\begin{array}{l}\text { Cadres du public, enseignants, } \\
\text { semi-professionnels }\end{array}$ & 9 & 8 \\
\hline Cadres du privé & 11 & 11 \\
\hline Propriétaires PME & 9 & 10 \\
\hline Cols blancs & 7 & 8 \\
\hline Superviseurs & 7 & 6 \\
\hline Propriétaires artisans & 9 & 10 \\
\hline Ouvriers artisans & 12 & 12 \\
\hline Ouvriers semi-spécialisés & 9 & 6 \\
\hline Ouvriers non-spécialisés & 10 & 5 \\
\hline Agriculteurs & 3 & 6 \\
\hline
\end{tabular}

Source: $\quad$ Les études universitaires au féminin (1982).

légère surreprésentation pour les femmes au niveau des collets blancs $(+3,7 \%)$ et une forte surreprésentation pour la même catégorie chez les hommes $(+16 \%)$. On peut donc conclure que les étudiants des deux sexes en service social ont une origine plus populaire que la population universitaire en général.

Dans son ensemble, la population étudiante universitaire actuelle en service social est très différente de celle qui fréquentait les écoles de service social au Québec au milieu de la décennie 1960. On se retrouve devant une transformation du modèle de recrutement de la population étudiante, en particulier de la population féminine en service social qui se traduit par un élargissement de la base sociale de recrutement qui provient maintenant majoritairement des classes populaires (60\%), alors que dans les années 1960 elle provenait d'origine sociale plus élevée (classe populaire: $25 \%{ }^{77}$ ).

On peut parler, à ce propos, d'une démocratisation des populations étudiantes en service social, d'un quasi-renouvellement qui s'apparente à une substitution. Cette nouvelle composition des

77. Les différences en pourcentage entre les années 1980 et 1960 sont respectivement pour les femmes en service social de $-15 \%$ de classe supérieure, de $+30 \%$ de classe ouvrière, et pour les hommes de $-3 \%$ de classe supérieure et de $+10 \%$ de classe ouvrière. 
effectifs étudiants, selon le sexe et l'origine sociale, traduit une modification du service social dans le champ occupationnel.

Cette reféminisation et l'origine plus populaire de ses agents semblent liées à la modification de la position du service social dans le champ occupationnel. L'institutionnalisation et la bureaucratisation des services sociaux semble avoir entraîné une dévaluation relative du statut occupationnel, ce qui a eu pour effet une féminisation du métier et un recrutement plus populaire. Ainsi tout semble se passer comme si le processus plus général de technocratisation des services sociaux avait entraîné une modification de la position occupée par le service social dans la structure des occupations, ce qui s'est répercuté sur le recrutement et l'origine sociale des futurs agents. On peut parler d'un processus social circulaire où la reféminisation du service social et l'élargissement de sa base sociale sont corrélatifs des modifications de sa position à l'intérieur du champ occupationnel.

La redéfinition féministe du service social apparue durant cette période, participe, dans ce contexte, à un «travail symbolique» transformant l'image sociale du métier ${ }^{78}$ afin d'assurer plus d'autonomie à l'intervention et un contrôle relatif sur un ensemble d'activités. En plus de se démarquer d'une fonctionnarisation de l'aide, cette redéfinition participe à l'élaboration d'un nouveau «marché de biens pédagogiques» dans la découverte, la construction de problèmes et de besoins peu reconnus socialement et de nouvelles pratiques d'intervention ou de nouveaux services pour $\mathrm{y}$ répondre. Le thème de l'oppression spécifique des femmes signale les problèmes urgents où il faut agir et les multiples regroupements de femmes qui s'établissent selon les diverses dimensions de cette oppression spécifique, grâce à l'action des intervenantes féministes, indiquent l'extension possible d'un marché de services sociaux: femmes en instance de séparation, femmes seules avec jeunes enfants, femmes en conflit avec leurs adolescents, femmes au-dessus de 40 ans, femmes qui veulent retourner sur le marché du travail, femmes chefs de famille monoparentale, femmes battues, etc. On arrive aussi à renouveler tant la perception que la clientèle des services sociaux. Dans chacun de ces groupes s'actualisent le travail de conscientisation, de partage du vécu, de solidarité et de lutte sociale contre les sources de l'oppression féminine. La critique des mécanismes d'aide professionnelle permet de contester la prétention d'agents professionnels à intervenir auprès de ces mêmes populations. Dans certains cas, le droit exclusif d'intervenir est explicitement revendiqué:

78. F. Muel-Dreyfus, Le métier d'éducateur (Paris, Minuit, 1983) et P. Bourdieu, «Classement, déclassement et reclassement», Actes de la recherche en sciences sociales, 24 (novembre 1978). 
Toutefois il est nettement préférable que l'intervention soit engagée par une femme (non sexiste et prioritairement féministe) [...] pour donner la priorité à une intervenante. 1) L'intervenante peut être un modèle féminin et un modèle d'affirmation, 2) Une femme se confie plus facilement à une autre femme, 3) La femme qui demande des services s'engage moins dans des jeux pour plaire à une intervenante [...] Il est également plus facile pour une femme de faire alliance et se solidariser avec une autre femme qui a reçu les mêmes messages sur sa condition et sur son devoir féminin ${ }^{79}$.

On peut émettre l'hypothèse que le discours féministe apparaît alors, sur l'ensemble des discours disponibles ${ }^{80}$, comme l'un de ceux qui permet au service social en voie de féminisation et de relégation institutionnelle, d'empêcher une dévaluation sociale qui s'exprime, entre autres, dans les nouvelles exigences technocratiques de déprofessionnalisation du poste. Ce discours assure «la défense de la rareté relative de l'identité sociale ${ }^{81}$ » en permettant d'invalider la prétention de concurrents à revendiquer un droit au poste à partir des seuls titres scolaires. Il permet d'ajouter au titre scolaire des dispositions éthiques et selon le sexe dont l'affirmation renforce une rareté relative par rapport à d'autres groupes concurrents, dépourvues soit du titre ou d'un engagement féministe.

\section{5 - ANALYSE ET INTERPRÉTATION}

Ce survol suscite plusieurs interprétations et questions que j'ai regroupées en trois thèmes.

1 - La professionnalisation/déprofessionnalisation du métier

2 - L'identité comme stratégie et éthique

3 - La question du contrôle social

79. G. Larouche, op. cit., 22.

80. P. Bourdieu, op. cit. Dans le jugement porté sur la clientèle comme sur les professionnels «concurrents», on retrouve une invalidation qui fonde la légitimité ou le droit à intervenir à la place des professionnels concurrents. La clientèle a «besoin» des services de l'intervenante féministe à cause des problèmes vécus qui sont formulés en termes d'incapacité, d'aliénation, d'oppression. Elles redeviennent compétentes, libérées, autonomes grâce à l'intervenante. Par ce discours on affirme, simultanément, les nouvelles catégories du problème, la nouvelle clientèle pour les services féministes et l'invalidité des autres services professionnels. Toute résistance ou critique de l'intervention sont réinterprétées dans le cadre du problème et servent de preuve à la nécessité ou à l'urgence d'intervenir.

81. Idem. 


\section{Tableau 5}

\section{Identités sociales du service social}

\begin{tabular}{|c|c|c|c|}
\hline Démotivation & Auxiliaire sociale & Travailleur social & Intervenante sociale \\
\hline Exigences & $\begin{array}{l}\text { Sentiment ou } \\
\text { vocation féminine }\end{array}$ & $\begin{array}{l}\text { Habilités } \\
\text { interpersonnelles et } \\
\text { maîtrise de soi }\end{array}$ & $\begin{array}{l}\text { Conscience } \\
\text { féministe }\end{array}$ \\
\hline $\begin{array}{l}\text { Attitude vis-à-vis } \\
\text { client }\end{array}$ & $\begin{array}{l}\text { Distance/condescen- } \\
\text { dance/moralisation }\end{array}$ & $\begin{array}{l}\text { Empathique et } \\
\text { contractuel }\end{array}$ & $\begin{array}{l}\text { Solidarité et } \\
\text { engagement }\end{array}$ \\
\hline $\begin{array}{l}\text { Attitude vis-à-vis } \\
\text { autorité }\end{array}$ & Respect & Négociation & $\begin{array}{l}\text { Critique/ } \\
\text { anti-hiérarchique }\end{array}$ \\
\hline $\begin{array}{l}\text { Attitude vis-à-vis } \\
\text { métier }\end{array}$ & Abnégation & $\begin{array}{l}\text { Distance au rôle } \\
\text { et neutralité }\end{array}$ & Engagement \\
\hline Aide & $\begin{array}{l}\text { Secours matériel et } \\
\text { aide morale }\end{array}$ & $\begin{array}{l}\text { Services } \\
\text { psychosociaux/ } \\
\text { relation d'aide }\end{array}$ & $\begin{array}{l}\text { Intervention } \\
\text { libération } \\
\text { conscientisation }\end{array}$ \\
\hline Famille & $\begin{array}{l}\text { Division stricte des } \\
\text { rôles et autorité } \\
\text { paternelle }\end{array}$ & $\begin{array}{l}\text { Egalisation des rôles } \\
\text { et autorité parentale }\end{array}$ & $\begin{array}{l}\text { Indifférenciation des } \\
\text { rôles et autonomie } \\
\text { féminine }\end{array}$ \\
\hline Morale & $\begin{array}{l}\text { Ascétique et } \\
\text { familialiste }\end{array}$ & $\begin{array}{l}\text { Permissive et } \\
\text { libérale }\end{array}$ & $\begin{array}{l}\text { Hédoniste, } \\
\text { expressive et } \\
\text { féministe }\end{array}$ \\
\hline Savoir & Pratique & Analytique & Critique \\
\hline Légitimité & Catholicisme social & $\begin{array}{l}\text { Savoir et formation } \\
\text { universitaire }\end{array}$ & Féminisme social \\
\hline Champ & Philanthropique & Professionnel & $\begin{array}{l}\text { Institutionnel ou } \\
\text { technocratique }\end{array}$ \\
\hline $\begin{array}{l}\text { Taux de } \\
\text { féminisation }\end{array}$ & $75 \%$ & $60 \%$ & $75 \%$ \\
\hline Origine sociale & $\begin{array}{l}\text { Ouvrière et } \\
\text { moyenne }\end{array}$ & $\begin{array}{l}\text { Moyenne et } \\
\text { supérieure }\end{array}$ & $\begin{array}{l}\text { Ouvrière et } \\
\text { moyenne }\end{array}$ \\
\hline $\begin{array}{l}\text { Origine sociale } \\
\text { (femmes) }\end{array}$ & $\begin{array}{l}\text { Ouvrière et } \\
\text { moyenne }\end{array}$ & $\begin{array}{l}\text { Moyenne et } \\
\text { supérieure }\end{array}$ & $\begin{array}{l}\text { Ouvrière et } \\
\text { moyenne }\end{array}$ \\
\hline $\begin{array}{l}\text { Origine sociale } \\
\text { (hommes) }\end{array}$ & Ouvrière & $\begin{array}{l}\text { Ouvrière et } \\
\text { moyenne }\end{array}$ & $\begin{array}{l}\text { Ouvrière et } \\
\text { moyenne }\end{array}$ \\
\hline
\end{tabular}

\section{1 - La professionnalisation/déprofessionnalisation du métier}

Cette triple identité ${ }^{82}$ du service social à trois moments différents rend problématique toute définition univoque de celui-ci. Ces identités

82. «La cristallisation d'une identité professionnelle sur un nom fait partie de ces opérations de façonnage, d'enregistrement et de négociation de l'identité». L. Thévenot, «À propos d'une définition des couches moyennes», Revue française de sociologie (avril-juin 1983): 318. 
apparaissent plus comme le résultat d'un processus persuasif et stratégique que comme l'affirmation d'attributs spécifiques ou la réalisation graduelle d'un modèle professionnel. La question de la légitimité de l'action reste présente et constante tout au long des périodes étudiées, légitimité qui demeure précaire, fragile et variable. Ainsi ce qui définissait le service social à une période est remis en question à la période suivante, voire considéré comme sa négation. Ainsi le sentiment féminin est vocationnel, constitutif au début de la pratique du service social, est perçu et interprété à la période professionnelle comme un obstacle à son exercice et comme source d'aliénation et l'oppression selon la perspective féministe. La perspective professionnelle est elle-même réinterprétée à cette dernière période comme arbitraire, sexiste et autoritaire. Ces multiples changements d'identité apparaissent pour une bonne part comme autant de figures d'un discours stratégique de revendication d'un statut social afin d'acquérir une autorité et une autonomie à l'intérieur du champ de l'action sociale.

L'histoire du service social apparait ici plus discontinue que continue, comme si un changement d'un élément amenait un changement systématique, interrelié et concomitant des autres éléments. Tout se passe comme si tout changement de la position d'un métier dans le champ social entraînait des changements concomitants des caractéristiques de ses agents tant aux plans du sexe, de l'origine sociale que des représentations du métier. On est alors amené à relativiser les analyses historiques qui étudient souvent l'évolution d'un métier en termes de professionnalisation, comme si l'acquisition des attributs professionnels (formation spécialisée sanctionnée par un diplôme, organisation corporative, code de déontologie, etc.) obéissaient à un processus autonome, naturel et inéluctable. Le processus de professionnalisation du service social apparaît comme transitoire et renvoie à la dynamique de la position du service social dans l'espace social.

La professionnalisation du service social traduit sa relative réussite à se faire reconnaître socialement comme profession et à s'assurer un lieu de formation et un marché en expansion, ce qui a favorisé une déféminisation de ses effectifs, une modification de son recrutement parallèle à une nouvelle image passant d'une identification féminine et vocationnelle à une définition méthodologique et professionnelle. Cette professionnalisation dépend en grande partie de l'État qui, le reconnaissant et le finançant comme service public, a favorisé son expansion et permis le développement de travailleurs sociaux qualifiés qui ont pu revendiquer le contrôle sur le champ des services sociaux. C'est aussi ce même développement de l'État qui a amené à sa relative déprofessionnalisation produisant ainsi une déqualification relative, ce 
qui n'a pas été sans effet sur sa reféminisation et le renouvellementsubstitution de ses effectifs quant à l'origine sociale, processus qui ont joué un rôle dans le renouvellement de l'identité, plus critique et féministe.

Dans ce contexte, la féminisation/reféminisation du service social ne nous semble pas une propriété secondaire de ce groupe, mais une manifestation importante des modifications sociales qui l'affectent. Ainsi la féminisation la plus forte du service social correspond aux deux périodes où ses activités sont plus faiblement reconnues. Il est à noter que durant ces deux périodes, vocationnelle et féministe, de féminisation/déprofessionnalisation, la légitimité du métier prend sa source dans des mouvements de réforme sociale, le catholicisme social en première période et le féminisme social en deuxième période, c'est-à-dire des références extérieures au métier lui-même. Est-ce à dire qu'aux deux périodes déprofessionnalisées, ces références aux mouvements de réforme sociale doivent être interprétées comme des stratégies symboliques pour accumuler un capital de légitimité nécessaire à son exercice ${ }^{83}$ ?

Peut-on aller jusqu'à affirmer que la définition vocationnelle et féminine est analogue à la définition engagée et féministe? Chaque définition ne renvoie-t-elle pas à la définition de la féminité dans les couches moyennes salariées à deux moments différents? $\mathrm{Ne}$ se retrouve-t-on pas, dans les deux cas, devant un métier qui permet d'actualiser sur le marché, un capital culturel lié au sexe, qualifié de féminin et féministe, fait de savoir-faire, de savoir-être acquis par socialisation diffuse ${ }^{84}$ ? Il y aurait entre le sentiment féminin et la conscience féministe des similitudes quant aux fonctions qu'ils rempliraient sur le marché des biens symboliques et économiques.

Cet investissement d'un capital culturel lié au sexe semble opérant pour les hommes et les femmes car on se retrouve, durant toute l'histoire du service social, devant des univers parallèles et polarisés où les domaines de la gestion, de la loi et du public sont masculins alors que les univers de l'enfance, de la famille, de la santé et du privé sont

83. Cette politisation de la société par des groupes professionnels a été aussi analysée pour les avocats. «On ne comprendrait pas le mouvement apparemment irrépressible des avocats vers la politique sans le rattacher au refus de la condition modeste qui leur était socialement assignée et aux chances, pour autant qu'ils sachent les reconnaître et les saisir, qu'offrait la politisation de la société». L. Karpik, op. cit., 126.

84. Cette socialisation ou ce vécu est toujours explicitement présentée comme base de la légitimité à intervenir. «C'est un cheminement personnel [...] c'était depuis des années en fait que je vivais comme femme des situations que toutes les femmes vivaient finalement, c'est-àdire de fille à la maison, ensuite de femme mariée, ensuite de femme divorcée, de femme chef de famille, d'étudiante à travers ça, travailleuse». 
féminins. Cette polarisation renverrait à une division sociale du travail entre les sexes qui est davantage horizontale en première et en deuxième période, c'est-à-dire selon les champs de pratique (enfancefamille-santé (femmes), judiciaire, communautaire (hommes) et devient verticale au milieu de la deuxième période (champs de pratique: femmes, et gestion: hommes).

\section{2 - L'identité comme stratégie éthique}

Ces redéfinitions successives du service social se présentent comme des stratégies symboliques d'une catégorie sociale pour contrôler certaines sphères d'activités ou comme «un travail symbolique sur l'image sociale du métier ${ }^{85} »$, afin de se positionner le plus avantageusement possible dans l'espace social ${ }^{86}$ en assurant «la défense de la rareté relative de l'identité sociale ${ }^{87}$ » et d'éviter le déclassement en se reclassant par des redéfinitions successives du poste.

Les redéfinitions successives d'identités sont parallèles à des redéfinitions de clientèles et de manières de traiter cette clientèle. Ainsi la redéfinition de la pauvreté, passant d'une conception morale et résiduelle à une conception psychosociale, est aussi la constitution d'un nouveau territoire d'intervention, d'un nouveau champ d'activités. Le même phénomène s'applique dans la redécouverte de la condition féminine. Ces redéfinitions participèrent d'un travail d'entrepreneurship moral ${ }^{88}$ dans l'identification des populations problèmes, des besoins de ces populations et la production ou la distribution des services pour répondre à ces besoins.

Le service social se présente aussi comme l'affirmation ou l'actualisation d'un système éthique, c'est-à-dire un ensemble systématique ou systématisé de normes qui s'exprime dans un ensemble de savoir-faire, savoir-être et de manières de les réaliser. Certaines de ces normes restent présentes tout au long des trois périodes; d'autres varient et se structurent différemment selon les divers modes d'organisation du champ de l'action sociale.

On retrouve certains thèmes invariants ou présents aux trois périodes. Celui de l'autonomie reste le plus prégnant, tant sur le plan de l'exercice du métier que de l'objectif à atteindre avec les clients. En première période ce thème prend une connotation morale dans la valo-

85. F. Muel-Dreyfus, «L'initiative privée, le terrain de l'éducation spécialisée», Actes de la recherche en sciences sociales (avril-juin 1980).

86. C. Bidou, Les aventuriers du quotidien (Paris, Presses universitaires de France, 1984).

87. P. Bourdieu, op. cit.

88. H. S. Becker, The Other Side (New York, Free Press, 1964). 
risation de l'effort, du «self-help», et dans la stigmatisation de la paresse. En deuxième période, il est repris explicitement, s'oppose à la dépendance et renvoie à la mobilisation des énergies personnelles et sociales; il est aussi réaffirmé, en troisième période, par rapport à l'oppression des femmes. La constance et la vigueur de ce thème provient peut-être de la position intermédiaire du service social dans le champ social, renforcé par la trajectoire sociale d'agents, provenant, dans une forte proportion, durant les trois périodes, de diverses factions des classes moyennes.

Les normes d'action véhiculées par le service social québécois varie aussi selon les divers champs de l'action sociale où il est inséré, qu'il soit philanthropique, professionnel ou technocratique ${ }^{89}$. L'éthique de la première période est traditionaliste et familialiste, centrée sur une représentation traditionnelle de la famille et de la différenciation entre les sexes selon une morale ascétique, valorisant l'ordre, la discipline et le respect de l'autorité. Ce système éthique s'intègre au champ philanthropique qui exige dans l'accomplissement des tâches pratiques de secours et d'aide, une motivation pratique et vocationnelle faite d'abnégation et de dévouement. La deuxième période privilégie une éthique davantage libérale, présentant une représentation plus «conjugaliste» de la famille tout en véhiculant une morale faite de maîtrise de soi, de contrôle des émotions, de juste milieu, de distance au rôle, d'aisance dans l'interaction sociale et de neutralité éthique, selon une attitude plus souple et négociatrice vis-àvis de l'autorité. Cet ensemble de valeurs correspond à des agents en position d'intermédiaire et répond aux exigences d'un champ professionnel qui privilégie la rationalisation de l'aide et du secours. La troisième période voit apparaître une éthique féministe centrée sur la libération de la femme selon une morale davantage permissive, voire hédoniste; une pratique faite de solidarité et de défense des droits de la clientèle et une attitude critique concernant diverses formes d'autorité familiale, institutionnelle ou professionnelle. Ces nouvelles

89. Certains autres thèmes sont présents au cours des trois périodes dont une certaine analyse «spiritualiste» des classes populaires. Dans la compréhension des problèmes de la "pauvreté», la dimension économique est souvent perçue comme secondaire par rapport à d'autres dimensions jugées principales comme la dimension morale, relationnelle, ou l'oppression sexiste. Tout se passe comme si les dimensions économiques et matérielles devraient être réinterprétées à l'intérieur de questions qui engagent une mobilisation "symbolique». Dans ce sens, la nécessité de mobiliser des énergies soit morales, interpersonnelles ou sociales qui renvoient à l'effort dans l'action qui se doit d'être pratique et faite avec un esprit de sérieux est, avec le thème de l'autonomie dont elle est une explication, l'une des principales dimensions de l'éthique des agents du service social qui gardent une relative stabilité aux trois périodes. Traduisent-elles leur position et trajectoire de classe qui ne doivent leur reproduction que par l'effort, l'esprit de sérieux et la mobilisation d'énergies ou de diverses formes «restreintes» de capital? 
normes s'affirment dans un champ social plus complexe et technocratique qui exige des agents déprofessionnalisés intervenant auprès de populations cibles spécifiques et délimitées ${ }^{90}$.

\section{3 - La question du contrôle social}

Sous certains aspects, le service social apparaît dans son évolution comme plus permissif si l'on s'en tient à l'évolution du rapport à la clientèle et plus directif si l'on tient compte du domaine et du degré d'intervention.

Ainsi à la première période, on se retrouve devant un service social condescendant qui favorise une implication minimale en gardant ses distances vis-à-vis une clientèle perçue sur le registre moral comme dangereuse ou paresseuse. À la deuxième période, le rapport se libéralise ou se démocratise; on insiste sur les capacités empathiques du travailleur social, de pouvoir ressentir ce que le client vit, tout en gardant une «distance bienveillante», favorisant un rapport contractuel avec la clientèle. À la troisième période, on privilégie un rapport davantage égalitaire, fait d'identification, de connivence et de solidarité.

Cette démocratisation de la relation avec la clientèle est concomitante à la modification du degré d'intervention auprès de la clientèle. À la première période, l'intervention est minimale faite de conseils moraux et d'aides financières ponctuelles, données avec parcimonie à partir des conditions strictes de nécessité et de moralité. Á la deuxième période, on se retrouve devant un système d'aide plus continu, basé sur la distribution de ressources psychosociales à «la demande du client». À la troisième période, les systèmes d'aide interviennent davantage dans la vie privée au nom de la violence conjugale et familiale avec pour objectif de modifier la structure des attitudes et des comportements allant jusqu'à modifier le rapport au corps. Quel est le sens de l'évolution divergente selon chacune de ces deux dimensions (relation à la clientèle - degré d'intervention)? L'efficacité

90. Il peut sembler paradoxal de lier de façon fonctionnelle l'intervention féministe et critique au champ technocratique. L'intervention féministe permet de redonner un nouveau sens à la pratique, à «ré-enchanter» le métier en se démarquant du travail banalisé de fonctionnaire, de réinvestir les acquis sociaux et culturels, de contrôler certaines conditions du marché de l'emploi et de fonctionner comme moyen d'accéder à une certaine visibilité sociale dans l'action de mobilisation des femmes. Cette intervention féministe permet, par son caractère critique, de se démarquer par rapport aux autres agents, de renforcer sa propre identité et d'assurer, par intégration conflictuelle, le fonctionnement stratifié et hiérarchisé des services sociaux et de participer à la division sociale du travail à l'intérieur de ce champ par la revendication d'un contrôle par les intervenantes féminines de la distribution de services sociaux à une populaiton «féminine» spécialisée. 
invisible résiderait-elle dans la diffusion de système de normes ou de mode de vie de classe moyenne? Tout semble se passer comme si la relation de pouvoir, inscrite dans la relation d'aide, ne pouvait s'exercer que niée par les agents eux-mêmes dans la revendication d'une égalité dans le contrat professionnel ou la solidarité féministe.

En résumé, on peut faire l'hypothèse que ces changements d'identité et du rapport au métier renvoient aux modifications de la position du service social dans le champ de l'action sociale et probablement dans le champ des classes moyennes, lui-même déterminé par les transformations de l'État et de ses appareils. Ces changements ou cette mobilité sociale du service social semble influer sur le recrutement social et la féminisation de ses agents ${ }^{91}$. On peut à cet égard construire une double représentation soit déterministe ou indéterministe de l'évolution du service social, selon que l'on insiste sur les déterminants des modifications des positions sociales du service social qui se répercutent sur le recrutement, la féminisation et la représentation ou selon que l'on insiste sur les stratégies symboliques des agents pour s'insérer dans le champ philanthropique, se construire un champ professionnel et se reclasser dans le champ technocratique sous le mode féministe.

91. Pour une autre analyse davantage fonctionnaliste du processus de féminisation et son articulation/adaptation aux autres changements qui touchent les professions, voir J. Collin, Changement d'ordonnance (Montréal, Éditions du Boréal, 1995). 